\title{
1 Philosophy and terminology
}

\author{
C.T. de Wit
}

\subsection{Systems, models and simulation}

Since the 1950s, engineers have paid much attention to the study of complex, dynamic systems. Their successes inspired biologists to apply similar techniques in their disciplines. The approach is characterized by the terms: system, model and simulation.

A system is a limited part of reality that contains interrelated elements. The totality of relations within the system is known as the 'system structure': both systems and models have a structure. A model is a simplified representation of a system. Simulation is the building of mathematical models and the study of their behaviour in reference to those of the systems.

There are many kinds of models. A simple mathematical model is the well-known relation between velocity and distance covered by a falling apple depending on the gravitational acceleration and the time from the moment of release. An example of a non-mathematical model is a map. This is a simplified representation of the earth's surface containing relevant information and it allows measurements of distances or areas. Depending on the objectives, a map will display railway lines, lines of equal rainfall or vegetation. A scale model of a ship in a towing tank enables measurements of its resistence in the water to help predict the behaviour of the real ship when this has been built.

It follows from the definition that a model is a system, but the reverse may also be true. A machine is a model of the conception of the engineer and it certainly performs worse than expected. And when an engineer applies simulation, he develops models that lie in between his conception and reality. The ultimate machine is in fact a model of his simulation model, which in its turn is a simplified representation of his mental conception.

Biological systems are not simplified representations of the conception of the biologist, even though current trends in agriculture might seem to be moving that way. Therefore, it may be that the approach that has been so successful in technology is not as useful in biology. There are, of course, ample examples of biological systems: a membrane, a cell, an organ, a plant, an animal, a field, a woodland and a lake. In this context, fields, woods, lakes, estuaries, pastures and all the rest have been given the general name of 'ecosystems'.

A system is a limited part of reality, so that a border has to be chosen. It is wise to select the boundary so that the system is isolated from its environment. This is hardly ever possible, but then it should be attempted to choose a border so that the environment might influence the system, but that the system affects the environment as little as possible. To this end, it is often essential to select a system that is larger 
than would seem necessary for the primary objective. Therefore, in ecological systems, the microclimate is often a part of the system, but most people willingly neglect -unjustly- the influence of the processes in the ecosystem on the macroclimate, that is considered to be a measurable environment not influenced by the ecosystem.

\subsection{Descriptive and explanatory models}

A file with data on an ecosystem might be called a model, but it is one of the most unclear and unusable kinds. Potential uses of the data may be formulated however, and then clarity may be introduced by a treatment of this data. This may result in maps that represent aspects of the ecosystem, or in statistical analysis which summarize some of the interrelations. If the time dimension is also taken into account during collection and treatment of the data, these models are no longer static but dynamie. However, those modets remain descripttie, showing the existence of relations between the elements of a system without any explanation. but. of course, this was not their original purpc.

However, explanatory models are possible in biology, because various levels of organization or knowledge are distinguished in this science, just like in all other natural sciences. These levels of knowledge distinguish themselves by the level of integration at which the processes occur. The different levels of integration may be classified, according to the size of the system, such as molecules, cell structures, cells, tissues, organs, individuals, populations, communities and ecosystems.

$\checkmark$ Explanatory models demand that research has to be carried out for at least two inte4 gration Tevels. The lower integration level will then be the explanatory level and the upper level is the one to be explained. In this way, one might attempt to derive the characteristics of membranes from the characteristics of the molecules of which they consist, or one might try to explain the processes in an ecosystem on the basis of knowledge of the behaviour and physiology of the constituent species. When knowledge at the explanatory level is sufficiently extensive and authoritative, and a model of the system to be explained is designed on this basis, it will not be necessary to test the model by comparing its results with those of the real system. For example, models for space travel are so good that the actual journey into space is unnecessary. But explanatory models in biology are frequently so inadequate that proof of their usefulness is necessary. Even if the results of the model correspond to the observations of the system being modelled, there is room for doubt regarding the correctness of the model. However, good agreement is still more the exception than the rule.

If there are discrepancies between the results of the model and reality, the model may be adjusted to obtain better agreement. Then, something that started as an explanatory model will degenerate into a descriptive model. The term 'degeneration' in this context does not mean that descriptive models are inferior to explanatory models. It is used here to emphasize that in this way inscrutable models are obtained with an unjustified pretention to explain something. This is the reason why many models made in ecological studies to date have done more harm than good.

The proper way of working is heuristic, by the path of methodical improvement. 
When unacceptable discrepancies between the model and the system are observed, it may be possible to determine by experiments with both the model and the system, which aspects of the model are suspicious. These aspects are then studied at the explanatory level. On basis of this new information, elements of the model may be replaced by improved versions, after which the results of the model and the reality may be compared once more.

Explanatory models can be static or dvnamic. An example of a static model is one in which the connection between respiration and growth of organisms is calculated on the basis of knowledge of the biochemical processes involved. Another example is a model in which the light distribution over the leaves of a vegetation is calculated from the canopy architecture, reflection and transmission of the leaves, solar position, and the brightness of the sky. The results of the calculations performed with these static models or the models themselves, often form a part of dynamic models. These dynamic models are simplified versions of dynamic systems, or in other words, systems that change with time. The development of these models and the study of their behaviour is frequently called 'simulation'.

\subsection{State-determined dynamic systems and models}

Simulation of ecological systems with an explanatory model is based on the asssumption that the state of every system at every moment can be quantitatively characterized and that changes in a system can be described by means of mathematical equations. This hypothesis leads to the formulation of state-determined dynamic models, in which state-, rate- and driving variables can be distinguished.

State variables are variables like the amounts of biomass, the number of animals, the amount of nitrogen or water in soil, plant or animal, etcetera.

Driving variables or forcing functions characterize the influence of external factors on the system, and are not influenced by the processes within the system. Their value must be monitored continuously. Examples are macro-meteorological variables such as rain, wind and radiation, or the supply of nutrients or migration of animals over the boundaries of the system. Depending on the system's limits, the same variables can be driving variables, or state or rate variables.

Rate variables indicate the rate at which the state variables change. Their values are determined by the state and driving variables according to rules that are based on knowledge of the physical, chemical and biological processes that take place in the system, and not on the basis of a statistical analysis of the behaviour of the system. This is the most important distinction between models that describe and models that attempt to explain.

After the calculation of all rate variables, these are used to calculate the state variables according to the scheme: state variable at time $t+\Delta t$ is equal to the state variable at time $t$ plus the rate at time $t$ multiplied by $\Delta t$. This procedure, called integration, gives the new values of the state variables, and the calculational procedure may be repeated. Obviously, modern calculators are indispensable here. Later in this book, various examples will be presented of applications in ecology.

Rates are not interdependent in state-determined systems: each rate depends at each 
moment on the value of state and driving variables and can therefore be calculated independent of any other rate. We can illustrate this mutual independence of rates by means of the following example. It will be clear that the rate of plant growth, as measured by the increase in weight of its structural tissues, is closely related to the net photosynthesis in the leaves. However, in an explanatory model of plant growth, this relation is the result of the simultaneous operation of various processes, and it is thus no direct relation. The rate of photosynthesis contributes to the amount of the plant's reserves and, also dependent upon other state variables, the growth rate of the various organs is connected with the amount of reserves. Although photosynthesis stops when it gets dark, growth does continue until the reserves have been exhausted, and sometimes even further, but then at the cost of previously formed structural tissues.

The number of state variables that can be distinguished in ecosystems is discouragingly large. This does not only involve plants, herbivores, carnivores and microorganisms, but also their various kinds, and of these the numbers, the size, the age, the development stage, etc. For plants, not only the weight and the surface area of the leaves are of importance, but also their nitrogen and mineral contents, their enzymes and other biochemical characteristics. It is possible to continue in this way, and this is the very reason why attempts at constructing models on the basis of a full knowledge of all the biological, physical and chemical processes are completely unrealistic. Models are nothing more than simplified representations of reality, and this simplification characterizes itself by the limited number of state variables that are taken into account.

Analogous to other scientific approaches, it is assumed that the number of state variables in a model is not only limited by the boundaries of the system to be studied, but also by a clear description of the objectives. Consequently, it can never be a reasonable objective of a model to fully comprehend an ecosystem, but if this is so, what might it then be? For every application of models or their construction, we must start by clearly describing the objective.

In agriculture the formulation of this objective is, initially, simple: to obtain insight into the relation between yield on the one hand and human effort on the other. This objective can be further detailed into questions regarding the relation between the addition of nitrogen and yield for well-defined boundary conditions. For every objective an optimum can be found for the number of state variables that should be included. Initially, the applicability of a model increases with an increasing number of state variables. But then the applicability decreases again because the inclusion of a new state variable diverts the attention from the state variables introduced previously because these were considered more important. The attempt at arranging the state variables in order of importance is very time-consuming, and many modelling efforts in ecology are sometimes explicitly, but more often implicitly,
geared towards this goal.

\subsection{The usefulness of ecological research using models}

The maintenance of the integrity and of options for developments of ecosystems, 
such as fields, woods, estuaries and the human society as a whole, is one of the greatest problems of society. The question is whether it is also a scientific problem. This will only be the case when the problem has not been solved and when it can be made acceptable on theoretical grounds that the problem can be solved. We need not worry about the former, and as far as solvability is concerned: precisely the existence of techniques that enable us to summarize knowledge in operative simulation models will inspire some people to an optimistic vision. But is this justified? Problems can only be solved when the solution can be falsified or, being rather more positive with regard to modelling, when models can be tested for their usefulness. To investigate the possibilities for this, it is useful to distinguish between verifiable and speculative models.

Verifiable models can only be created from repeatable or recurring systems. Examples of repeatable systems are continuous cultures of bacteria, farms and industrial processes. Recurring systems appear to the observer at different places at the same time in different stages. Examples are: stars, individuals of a species and ecological systems whose development in time is controlled by strong negative feedbacks, such as peat bogs. Of these latter ecosystems no two will ever be the same, but this does not imply that their models might not be identical: after all, a model remains a simplified representation of reality. It is clear that experiments can always be carried out with repeatable systems, but that recurring systems are not or less accessible to experimentation. Ecology develops from a descriptive science to a science in which experiments are carried out with repeatable and recurring systems. The latter is justified because the chance of disturbances is small due to the existence of negative feedbacks in these systems, and because there are many of them. Systemanalytic research and simulation should be limited to repeatable and recurring systems to even a greater extent than the experiments, because the knowledge of the processes that occur is still so limited that verification of models is needed.

Besides repeatable and recurring systems, unique systems exist. Examples of these are climatic systems and systems determined by geographical circumstances such as the Oosterschelde (Eastern estuary of the river Schelde), the Waddenzee (Wadden Sea to the north of the Netherlands), the world itself and human society, as well as ecosystems whose development is only controlled by weak negative feedbacks within narrow limits, so that the originally identical systems may diverge in space and time. Evolution itself is an example of such a system and consequently also breeding in agriculture. Precisely because the breeding process is irreversible, it is only justified when measures are taken to conserve the gene pool. Models of unique systems are speculative models, since they cannot be tested for their usefulness. After all, analysis and testing should apply to completely independent systems if the results of the test are to lead to the possibility of rejecting the model. Of course, unique systems possess recurring elements and these can be isolated and thus made accessible for experiments and the construction of models. Therefore, unique systems can be partially analysed and sometimes experiments can be performed within these systems. The significance of observation and experimentation should then be weighed against the risk of disturbing the system; in case of doubt no experiments should be done. 
Therefore, genetic manipulation should be carried out with the greatest care.

Speculative models cannot be verified but they can more or less be trusted. The confidence in these models will grow when analogous methods of analysing repeatable and recurring systems lead to the formulation of verifiable models with useful results. The confidence in models of unique systems whose behaviour is controlled by physical phenomena might even be quite large. Nobody need seriously doubt the calculations regarding the relation between the height of the dikes and the chance of flooding in the Netherlands, but when flooding occurs, confidence will have decreased dramatically.

In ecology, useful models have only been constructed of relatively simple systems, and according to some, only of those systems that also could have been understood without the use of the advanced methods of systems analysis and simulation. Therefore, confidence in speculative models of unique systems is justifiable small. The tragic is that, scientifically speaking, ecologists are only able to make statements on a growing number of repeatable and recurring systems, but that society is demanding statements on the development of the unique systems. Meeting society's requirements can therefore easily lead to plainly fraudulent comments. Many ecologists are well aware of this and refrain from making statements, but this then results in this blank patch in our knowledge being filled in irresponsibly by so-called instant-ecologists.

'Global models' with any perspective of being useful are more complicated than models of those ecosystems whose usefulness has been demonstrated. Indeed, these models should include knowledge from many different scientific disciplines, and in some of the relevant scientific disciplines, this knowledge is significantly smaller than for those of the physical, chemical and biological processes that will play a role in simple ecosystems. This will mean that the basis for confidence will be missing and in many cases will not even be laid. Consequently, 'global' models remain unusable instruments in the preparation of policy decisions. Therefore, the question is whether research efforts should be made to analyse 'global' systems that may result in speculative, interdisciplinary models. Nevertheless, the answer is in the affirmative. The basis for eventual confidence can be laid by comparative research of verifiable and speculative models. Systems analysis and modelling are the only developing interdisciplinary professional fields that enable us to integrate and oversee our incomplete knowledge, and it is pointless to throw away half-worn-out shoes before new ones have been designed. And long before speculative, interdisciplinary models can be used to support governmental decisions in society, these kinds of models can be used as instruments of research policy in order to indicate research priorities.

Criteria can be formulated to which speculative, interdisciplinary models and considerations must comply in order to contribute to scientific development. This can result in recommendations for research that become increasingly important when more models are evaluated in a comparable manner. Possible criteria are:

- The objectives of the model must be well-described.

- Based on the objectives it should be argued which disciplines are to contribute to the development of the model, and to what extent.

- The contributions from the various disciplines should be reasonably scientifically 
reliable.

- Verifiable parts of the model should indeed be tested.

- Claims to usefulness should be critically evaluated.

- The model must be sound; this means that it should be known which suppositions have been incorporated, and this should be done in such manner that the consequences of other suppositions can be determined within the framework of the model's objectives.

- The model must be used soundly. This means that the designers will have investigated and commented on the consequences of a reasonable number of suppositions, and not only those suppositions which might confirm presuppositions on the results.

- The model must be clear and others than the designers should be able to use it. 